\title{
NON-AUTONOMOUS IMPULSIVE CAUCHY PROBLEMS OF PARABOLIC TYPE INVOLVING NONLOCAL INITIAL CONDITIONS
}

\author{
RONG-NIAN WANG, KHALIL EZZINBI AND PENG-XIAN ZHU \\ Communicated by Stig-Olof Londen
}

\begin{abstract}
We consider a nonautonomous impulsive Cauchy problem of parabolic type involving a nonlocal initial condition in a Banach space $X$, where the operators in linear part (possibly unbounded) depend on time $t$ and generate an evolution family. New existence theorems of mild solutions to such a problem, in the absence of compactness and Lipschitz continuity of the impulsive item and nonlocal item, are established. The non-autonomous impulsive Cauchy problem of neutral type with nonlocal initial condition is also considered. Comparisons with available literature are also given. Finally, as a sample of application, these results are applied to a system of partial differential equations with impulsive condition and nonlocal initial condition. Our results essentially extend some existing results in this area.
\end{abstract}

1. Introduction. The dynamics of evolving processes is often subjected to abrupt changes at certain moments such as shocks, harvesting and natural disasters. Often these short-term perturbations are treated as having acted instantaneously or in the form of "impulses" (see [4]). One of the emerging branches of the study associated with "impulses" is the theory of impulsive differential equations (inclusions). It is significant to study this class of equations (inclusions), because, in this way, a large class of physical processes in population biology, the diffusion of chemicals, the spread of heat, the radiation of electromagnetic waves, and so forth, can be analyzed. These processes usually have short-time

2010 AMS Mathematics subject classification. Primary 65J08, Secondary 34A37, 35R12.

Keywords and phrases. Non-autonomous evolution equation, nonlocal initial condition, impulsive condition, parabolicity condition, neutral type, mild solution.

The first author wishes to acknowledge support from the NNSF of China (Nos. 11101202, 11101203).

The first author is the corresponding author.

Received by the editors on March 4, 2013, and in revised form on June 29, 2013. 
perturbations during their evolution. The perturbations are performed discretely, and their duration is negligible in comparison with the total duration of the processes. Some advances concerning this topic can be found, for instance, in the monographs of Bainov and Simeonov [4], Benchohra et al. [7], Lakshmikantham et al. [20], Samoilenko and Perestyuk [29] and the papers of Ahmed [1, 2], Cardinali and Rubbioni [9], Hernández and Aki [19], Liu [22], Liu and Willms [24], Nieto and O'Regan [26] and Rogovchenko [28].

In particular, stimulated by the observation that nonlocal initial conditions are more realistic than usual ones in treating physical problems, the study of impulsive differential equations (inclusions) with nonlocal initial conditions has been investigated to a large extent; for significant works along this line, we refer to, e.g., $[\mathbf{3}, \mathbf{7}, \mathbf{1 3}, \mathbf{1 4}, \mathbf{1 7}]$ and the references therein. Please see $[\mathbf{8}, \mathbf{1 0}, \mathbf{1 1}, \mathbf{3 0}]$ for more detailed information related to the importance of nonlocal initial conditions in applications.

Let $X$ be a Banach space with norm $\|\cdot\|$. We consider the nonautonomous impulsive Cauchy problem of parabolic type involving nonlocal initial condition

$$
\left\{\begin{array}{l}
u^{\prime}(t)=A(t) u(t)+F(t, u(t)), \quad t \in[0, T] \backslash\left\{t_{1}, \ldots, t_{n}\right\}, \\
\Delta u\left(t_{i}\right)=I_{i}\left(u\left(t_{i}\right)\right), \quad i=1, \ldots, n, \\
u(0)=H(u),
\end{array}\right.
$$

in $X$, where $T>0,(A(t))_{t \in[0, T]}$ is a family of (possibly unbounded) linear operators depending on time and having the domains $(D(A(t)))_{t \in[0, T]}$, $0<t_{1}<t_{2}<\cdots<t_{n}<T$ are pre-fixed numbers, $\Delta u\left(t_{i}\right)$ represents the jump of the function $u$ at $t_{i}$, which is defined by $u\left(t_{i}^{+}\right)-u\left(t_{i}^{-}\right)$, where $u\left(t_{i}^{+}\right)=\lim _{h \rightarrow 0^{+}} u\left(t_{i}+h\right)$ and $u\left(t_{i}^{-}\right)=\lim _{h \rightarrow 0^{-}} u\left(t_{i}+h\right)$ denote respectively the right and left limits of $u(t)$ at $t=t_{i}$, and $F, H, I_{i}$ $(i=1, \ldots, n)$ are appropriate functions to be specified later. As can be seen, $H$ constitutes a nonlocal condition and $\Delta u\left(t_{i}\right)=u\left(t_{i}^{+}\right)-u\left(t_{i}^{-}\right)$ constitutes an impulsive condition.

As usual, the solution $t \rightarrow u(t)$ with the points of discontinuity at the moments $t_{i}(i=1, \ldots, n)$ follows that $u\left(t_{i}\right)=u\left(t_{i}^{-}\right)$, that is, at which it is continuous from the left.

We mention that in [21], Liang et al. studied the existence and uniqueness of mild and classical solutions to the Cauchy problem (1.1) in the autonomous case (i.e., $A(t) \equiv A$ ), with nonlocal item $H$ being 
Lipschitz continuous, compact, or not Lipschitz continuous and not compact, and impulsive item $I_{i}$ being Lipschitz continuous or compact.

In the present paper, we will combine this earlier work and extend the study to the Cauchy problem (1.1), which is more general than those in many previous publications. New results concerning the existence of mild solutions to the Cauchy problem (1.1), in the absence of compactness and Lipschitz continuity of the impulsive and nonlocal items, are established by using evolution family and the approximating technique. Then, we treat the generalization to the Cauchy problem (1.1) in the form

$$
\left\{\begin{array}{l}
\frac{d}{d t}[u(t)-g(t, u(t))]=A(t) u(t)+F(t, u(t)), \quad t \in[0, T] \backslash\left\{t_{1}, \ldots, t_{n}\right\}, \\
\Delta u\left(t_{i}\right)=I_{i}\left(u\left(t_{i}\right)\right), \quad i=1, \ldots, n, \\
u(0)=H(u),
\end{array}\right.
$$

where $g$ is an appropriate function to be specified later.

Let us point out that the work of this paper has two wedges; on the one hand, we will extend the study of autonomous impulsive Cauchy problems with nonlocal initial conditions to non-autonomous ones. On the other hand, we will obtain the existence theorems of mild solutions to the Cauchy problems (1.1) and (1.2) under weaker conditions on the impulsive and nonlocal items. The results obtained in this paper are generalizations of related results (see Remarks 1.1 and 1.2 below). Moreover, even for corresponding abstract impulsive Cauchy problems without nonlocal initial conditions, the results here are new.

Remark 1.1. As the reader can see, the hypotheses on the impulsive and nonlocal items in our theorems are reasonably weak and different from those in many previous papers such as $[\mathbf{3}, \mathbf{7}]$, and the proofs provided are concise.

Remark 1.2. One will see that Theorem 3.8 below essentially extends the main results of the previous research in several ways; as far as the mild solution of Cauchy problems (1.1) is concerned, by dropping the compactness and Lipschitz continuity of the impulsive item from the hypotheses. This distinguishes the present paper from earlier works on impulsive Cauchy problems. 
Remark 1.3. Note that the techniques in the proofs of our theorems are essentially different from those used in [15].

2. Preliminaries. In this section, we introduce some notation, establish some conventions and describe some results which are essential tools in later sections.

Let $C([c, d] ; X)$ for $-\infty<c<d<+\infty$ be the Banach space of all continuous functions from $[c, d]$ into $X$ with uniform norm topology. $\mathscr{L}(X)$ stands for the Banach space of all bounded linear operators from $X$ to $X$ equipped with its natural topology.

Write

$$
J_{0}=\left[0, t_{1}\right], \quad J_{i}=\left(t_{i}, t_{i+1}\right], \quad i=1, \ldots, n,
$$

with $t_{0}=0, t_{n+1}=T$, and let $u_{i}$ be the restriction of a function $u$ to $J_{i}(i=0,1, \ldots, n)$. Consider the set of functions

$$
\begin{aligned}
& P C([0, T] ; X)=\left\{u:[0, T] \rightarrow X ; u_{i} \in C\left(J_{i} ; X\right), i=0,1, \ldots, n,\right. \\
& \text { and } u\left(t_{i}^{+}\right) \text {and } u\left(t_{i}^{-}\right) \text {exist and satisfy } \\
& \left.\qquad u\left(t_{i}\right)=u\left(t_{i}^{-}\right) \text {for } i=1, \ldots, n\right\} .
\end{aligned}
$$

Endowed with the norm

$$
\|u\|_{P C}=\max \left\{\sup _{t \in J_{i}}\left\|u_{i}(t)\right\| ; \quad i=0,1, \ldots, n\right\}
$$

it is easy to show that $P C([0, T] ; X)$ is a Banach space (see $[\mathbf{1 8}]$ ).

Let $r$ be a finite positive constant, and put

$$
\Omega_{r}=\{u \in P C([0, T] ; X) ;\|u(t)\| \leq r, \quad \text { for all } t \in[0, T]\},
$$

which is a convex closed subset of $P C([0, T] ; X)$.

For a set $B \subset P C([0, T] ; X)$, we denote by

$$
\begin{gathered}
\left.B\right|_{\bar{J}_{i}}=\left\{u \in C\left(\bar{J}_{i} ; X\right): u\left(t_{i}\right)=v\left(t_{i}^{+}\right), u(t)=v(t), t \in J_{i}, v \in B\right\}, \\
i=0,1, \ldots, n .
\end{gathered}
$$


We present the following lemma, which is useful for our further study in this work.

Lemma 2.1. $A$ set $B \subset P C([0, T] ; X)$ is precompact in $P C([0, T] ; X)$ if and only if the set $\left.B\right|_{\bar{J}_{i}}$ is precompact in $C\left(\bar{J}_{i} ; X\right)$ for each $i=$ $0,1, \ldots, n$.

Definition 2.2. An operator family $\{U(t, s)\}_{0 \leq s \leq t \leq T} \subset \mathscr{L}(X)$ on $X$ is called a (strongly continuous) evolution family if

(1) $U(t, r) U(r, s)=U(t, s), U(t, t)=I$ for $0 \leq s \leq r \leq t \leq T$.

(2) The map $(t, s) \mapsto U(t, s)$ is strongly continuous for $0 \leq s \leq t \leq T$.

In this paper $\{A(t)\}_{t \in[0, T]}$ is assumed to be a family of linear operators defined in $X$. Frow now on, Hypotheses (a)-(b) (parabolicity conditions) below will be assumed throughout.

(a) The domain $D(A(t))=D$ of $A(t), 0 \leq t \leq T$, is dense in $X$ and independent of $t$.

(b) For every $t \in[0, T]$ and $\lambda \in \mathbf{C}$ with $\operatorname{Re} \lambda \leq 0$, the resolvent $(\lambda+A(t))^{-1}$ exists in $\mathscr{L}(X)$ and satisfies

$$
\left\|(\lambda+A(t))^{-1}\right\|_{\mathscr{L}(X)} \leq \frac{M_{0}}{1+|\lambda|}, \quad t \in[0, T], \operatorname{Re} \lambda \leq 0
$$

for a constant $M_{0}$.

(c) There are constants $\alpha \in(0,1]$ and $M_{1}$ such that

$$
\left\|(A(t)-A(s)) A(r)^{-1}\right\|_{\mathscr{L}(X)} \leq M_{1}|t-s|^{\alpha}, \quad t, s, r \in[0, T] .
$$

Under the Hypotheses (a)-(c), there is a unique evolution family $\{U(t, s)\}_{0 \leq s \leq t \leq T}$ satisfying:

(i) $\|U(t, s)\|_{\mathscr{L}(X)} \leq M$ for $0 \leq s \leq t \leq T$.

(ii) For $0 \leq s<t \leq T, U(t, s): X \rightarrow D$ and $t \rightarrow U(t, s)$ is strongly differentiable in $X$. The derivative $\left(\partial / \partial_{t}\right) U(t, s) \in \mathscr{L}(X)$, and it 
is strongly continuous on $0 \leq s<t \leq T$. Moreover,

$$
\begin{aligned}
& \frac{\partial U(t, s)}{\partial t}-A(t) U(t, s)=0 \quad \text { for } 0 \leq s<t \leq T \\
& \left\|\frac{\partial U(t, s)}{\partial t}\right\|_{\mathscr{L}(X)}=\|A(t) U(t, s)\|_{\mathscr{L}(X)} \leq \frac{M^{\prime}}{t-s} \quad \text { for } 0 \leq s<t \leq T, \\
& \left\|A(t) U(t, s) A(s)^{-1}\right\|_{\mathscr{L}(X)} \leq M^{\prime \prime} \quad \text { for } 0 \leq s \leq t \leq T .
\end{aligned}
$$

Here $M, M^{\prime}$ and $M^{\prime \prime}$ are positive constants.

(iii) For every $u \in D$ and $t \in(0, T], U(t, s) u$ is differentiable with respect to $s$ on $0 \leq s<t \leq T$ and

$$
\frac{\partial^{+} U(t, s) u}{\partial s}=U(t, s) A(s) u \text {. }
$$

In this case, $\{U(t, s)\}_{0 \leq s \leq t \leq T}$ is called the evolution family associated with $\{A(t)\}_{t \in[0, T]}$. "Evolution family" is also called evolution system, evolution operator, evolution process, propagator or fundamental solution. More details can be found in, e.g., [12, 23, 27, 31].

The considerations of this paper also need the following result.

Lemma 2.3. (Krasnoselskii's fixed point theorem). Let $E$ be a Banach space, $B$ a bounded closed and convex subset of $E$, and let $F_{1}, F_{2}$ be maps of $B$ into $E$ such that $F_{1} x+F_{2} y \in B$ for every pair $x, y \in B$. If $F_{1}$ is a contraction and $F_{2}$ is completely continuous, then the equation $F_{1} x+F_{2} x=x$ has a solution on $B$.

Lemma 2.3 is classical and can be found in many books.

3. Main results. The present section is devoted to the study of mild solutions to Cauchy problem (1.1). We start with the definition of mild solutions.

Definition 3.1. A solution $u \in P C([0, T] ; X)$ of the impulsive integral equation

$$
\begin{aligned}
u(t)= & U(t, 0) H(u)+\int_{0}^{t} U(t, s) F(s, u(s)) \mathrm{d} s \\
& +\sum_{0<t_{i}<t} U\left(t, t_{i}\right) I_{i}\left(u\left(t_{i}^{-}\right)\right), \quad t \in[0, T],
\end{aligned}
$$


is called a mild solution of Cauchy problem (1.1).

Let us first introduce our basic assumptions:

$\left(\mathrm{H}_{1}\right)$ The evolution family $\{U(t, s)\}_{0 \leq s \leq t \leq T}$ is compact, specifically, $U(t, s)$ maps bounded subsets of $X$ into precompact subsets of $X$ for all $0 \leq s<t \leq T$.

$\left(\mathrm{H}_{2}\right) \quad F:[0, T] \times X \mapsto X$ is a Carathéodory function; there exists a function $f_{r}(\cdot) \in L^{1}\left(0, T ; \mathbf{R}^{+}\right)$such that for almost every $t \in[0, T]$ and all $u \in X$ satisfying $\|u\| \leq r$, $\|F(t, u)\| \leq f_{r}(t), \quad$ and $\quad \liminf _{r \rightarrow+\infty} \frac{\left\|f_{r}\right\|_{L^{1}(0, T)}}{r}=\sigma<\infty$.

$\left(\mathrm{H}_{3}\right)$ (i) $H: P C([0, T] ; X) \mapsto X$ is continuous; there exists a nondecreasing function $\Phi: \mathbf{R}^{+} \rightarrow \mathbf{R}^{+}$such that, for all $u \in \Omega_{r}$,

$$
\|H(u)\| \leq \Phi(r), \quad \text { and } \quad \liminf _{r \rightarrow+\infty} \frac{\Phi(r)}{r}=\mu<\infty .
$$

(ii) There is an $\eta \in\left(0, t_{1}\right)$ such that, for any $u, w \in P C([0, T] ; X)$ satisfying $u(t)=w(t)(t \in[\eta, T]), H(u)=H(w)$.

$\left(\mathrm{H}_{4}\right)$ For every $i=1, \ldots, n, I_{i}: X \mapsto X$ is continuous, there exists a nondecreasing function $\Psi_{i}: \mathbf{R}^{+} \rightarrow \mathbf{R}^{+}$such that, for all $u \in X$ satisfying $\|u\| \leq r$,

$$
\left\|I_{i}(u)\right\| \leq \Psi_{i}(r), \quad \text { and } \quad \liminf _{r \rightarrow+\infty} \frac{\Psi_{i}(r)}{r}=\gamma_{i}<\infty
$$

Remark 3.2. Let us note that if, for each $t \in[0, T]$ and some $\lambda \in \rho(A(t))$ (the resolvent set of $A(t)$ ), the resolvent $R(\lambda, A(t)$ ) is a compact operator, then $U(t, s)$ is a compact operator whenever $t>s$ (see [16, Proposition 2.1]). Moreover, the compactness of $U(t, s)$ for $t>s$ implies the continuity in uniform operator topology.

Remark 3.3. Assumption $\left(\mathrm{H}_{3}\right)$ (ii) is the case when the values of the solution $u(t)$ for $t$ near zero do not affect $H(u)$. A case in point was presented by Deng [11], where the operator $H$ is given as follows: $H(u)=\sum_{i=1}^{p} C_{i} u\left(s_{i}\right)$, where $C_{i}(i=1, \ldots, p)$ are given constants and $0<s_{1}<\cdots<s_{p-1}<s_{p}<+\infty(p \in \mathbf{N})$, which is used to describe the diffusion phenomenon of a small amount of gas in a transparent tube. 
Before proving the main theorems, we first present some lemmas.

Lemma 3.4. Under hypotheses $\left(\mathrm{H}_{1}\right)$ and $\left(\mathrm{H}_{2}\right)$, the operator $\Gamma^{1}: \Omega_{r} \mapsto$ $P C([0, T] ; X)$, defined by

$$
\left(\Gamma^{1} u\right)(t)=\int_{0}^{t} U(t, s) F(s, u(s)) \mathrm{d} s, \quad u \in \Omega_{r}, t \in[0, T],
$$

is compact.

Proof. A standard argument, taking into account hypotheses $\left(\mathrm{H}_{1}\right)$ and $\left(\mathrm{H}_{2}\right)$, shows that the assertion of the lemma remains true (see also the proof of Lemma 3.6 below). Here, we omit the details.

Write $\Theta:=\left\{m ; m \in \mathbf{N}^{+}\right.$and $\left.m T \geq 1\right\}$. It is clear that

$$
\|U(t, s)\|_{\mathscr{L}(X)} \leq M \quad \text { for all } 0 \leq s \leq t \leq \frac{1}{m}(m \in \Theta) .
$$

Now, consider, for each $m \in \Theta$, an operator $\Gamma_{m}$ on $P C(0, T ; X)$ defined by

$$
\begin{aligned}
\left(\Gamma_{m} u\right)(t)= & U(t, 0) U\left(\frac{1}{m}, 0\right) H(u)+\int_{0}^{t} U(t, s) F(s, u(s)) \mathrm{d} s \\
& +\sum_{0<t_{i}<t} U\left(t, t_{i}\right) U\left(\frac{1}{m}, 0\right) I_{i}\left(u\left(t_{i}^{-}\right)\right), \quad t \in[0, T] .
\end{aligned}
$$

Lemma 3.5. Let hypotheses $\left(\mathrm{H}_{1}\right)-\left(\mathrm{H}_{4}\right)$ hold, except for $\left(\mathrm{H}_{3}\right)$ (ii). Then $\Gamma_{m}$ has at least one fixed point $u_{m} \in P C(0, T ; X)$ for each $m \in \Theta$, provided that

$$
M \sigma+M^{2}\left(\mu+\sum_{i=1}^{n} \gamma_{i}\right)<1
$$

Proof. Fix $m \in \Theta$. From our hypotheses on $F, H$ and $I_{i}(i=$ $1, \ldots, n)$ and $(3.1)$, it is easy to see that $\Gamma_{m}$, mapping $P C(0, T ; X)$ into itself, is well defined and there exists a $k_{0}>0$ such that

$$
M\left\|f_{k_{0}}\right\|_{L^{1}(0, T)}+M^{2}\left(\Phi\left(k_{0}\right)+\sum_{i=1}^{n} \Psi_{i}\left(k_{0}\right)\right) \leq k_{0},
$$


from which we see that, for $t \in[0, T]$ and $u \in \Omega_{k_{0}}$,

$$
\begin{aligned}
\left\|\left(\Gamma_{m} u\right)(t)\right\| \leq & \left\|U(t, 0) U\left(\frac{1}{m}, 0\right)\right\|_{\mathscr{L}(X)}\|H(u)\| \\
& +\int_{0}^{t}\|U(t, s)\| \mathscr{L}(X)\|F(s, u(s))\| \mathrm{d} s \\
& +\sum_{0<t_{i}<t}\left\|U\left(t, t_{i}\right) U\left(\frac{1}{m}, 0\right)\right\|_{\mathscr{L}(X)}\left\|I_{i}\left(u\left(t_{i}^{-}\right)\right)\right\| \\
\leq & M^{2} \Phi\left(k_{0}\right)+M \int_{0}^{t} f_{k_{0}}(s) \mathrm{d} s+M^{2} \sum_{i=1}^{n} \Psi_{i}\left(k_{0}\right) \\
\leq & k_{0} .
\end{aligned}
$$

This proves that $\Gamma_{m}$ maps $\Omega_{k_{0}}$ into itself.

Let $\left\{u_{q}\right\}_{q=1}^{\infty} \subset \Omega_{k_{0}}$ be a sequence such that $u_{q} \rightarrow u$ as $q \rightarrow \infty$ in $P C(0, T ; X)$. Observe, by the continuity of $H, I_{i}(i=1, \ldots, n)$, and $F$ with respect to the second argument, that for each $t \in[0, T]$,

$$
\begin{aligned}
\left\|\left(\Gamma_{m} u_{q}\right)(t)-\left(\Gamma_{m} u\right)(t)\right\| \leq & M^{2}\left\|H\left(u_{q}\right)-H(u)\right\| \\
& +M \int_{0}^{t}\left\|F\left(s, u_{q}(s)\right)-F(s, u(s))\right\| \mathrm{d} s \\
& +M^{2} \sum_{i=1}^{n}\left\|I_{i}\left(u_{q}\left(t_{i}^{-}\right)\right)-I_{i}\left(u\left(t_{i}^{-}\right)\right)\right\| \\
& \longrightarrow 0, \quad \text { as } q \rightarrow \infty,
\end{aligned}
$$

due to the Lebesgue dominated convergence theorem. That is,

$$
\left\|\Gamma_{m} u_{q}-\Gamma_{m} u\right\|_{P C} \longrightarrow 0, \quad \text { as } q \rightarrow \infty .
$$

Accordingly, $\Gamma_{m}$ is continuous on $\Omega_{k_{0}}$.

Next, to be able to apply Schauder's second fixed point theorem to obtain a fixed point of $\Gamma_{m}$, we need to prove that $\Gamma_{m}$ is compact on $\Omega_{k_{0}}$. Since $H\left(\Omega_{k_{0}}\right)$ is bounded in $X$ in view of $\left(\mathrm{H}_{3}\right)$ (i) and $U((1 / m), 0)$ is compact in $X$ in view of $\left(\mathrm{H}_{1}\right)$, we justify that, for each $t \in[0, T]$,

$$
\left\{U(t, 0) U\left(\frac{1}{m}, 0\right) H(u) ; u \in \Omega_{k_{0}}\right\} \text { is precompact in } X,
$$

by the boundedness of $U(t, 0)(t \in[0, T])$ and, for $0 \leq s \leq t \leq T$, 


$$
\begin{aligned}
\left\|U(t, 0) U\left(\frac{1}{m}, 0\right) H(u)-U(s, 0) U\left(\frac{1}{m}, 0\right) H(u)\right\| & \\
=\left\|[U(t, 0)-U(s, 0)] U\left(\frac{1}{m}, 0\right) H(u)\right\| & \stackrel{\longrightarrow}{\longrightarrow} \text { as } t \rightarrow s,
\end{aligned}
$$

by the strong continuity of $U(t, s)$ and the compactness of $U((1 / m), 0)$ $H(u)$ in $X$. Thus, we verify, with the aid of Arzela-Ascoli's theorem, that

$$
U(t, 0) U\left(\frac{1}{m}, 0\right) H(\cdot),
$$

mapping $\Omega_{k_{0}}$ into $P C([0, T] ; X)$, is compact.

The same idea can be used to prove that

$$
\sum_{0<t_{i}<t} U\left(t, t_{i}\right) U\left(\frac{1}{m}, 0\right) I_{i}\left(\cdot\left(t_{i}^{-}\right)\right),
$$

mapping $\Omega_{k_{0}}$ into $P C([0, T] ; X)$, is compact.

In fact, this can be seen from Lemma 2.1 and the observations that, for every $t \in \bar{J}_{i}(i=1, \ldots, n)$,

$$
\left\{U\left(t, t_{i}\right) U\left(\frac{1}{m}, 0\right) I_{i}\left(u\left(t_{i}^{-}\right)\right) ; u \in \Omega_{k_{0}}\right\} \text { is precompact in } X
$$

due to the compactness of $U((1 / m), 0)$, and for $t_{i} \leq s \leq t \leq t_{i+1}$ $(i=1, \ldots, n)$,

$$
\begin{gathered}
\left\|U\left(t, t_{i}\right) U\left(\frac{1}{m}, 0\right) I_{i}\left(u\left(t_{i}^{-}\right)\right)-U\left(s, t_{i}\right) U\left(\frac{1}{m}, 0\right) I_{i}\left(u\left(t_{i}^{-}\right)\right)\right\| \\
=\left\|\left[U\left(t, t_{i}\right)-U\left(s, t_{i}\right)\right] U\left(\frac{1}{m}, 0\right) I_{i}\left(u\left(t_{i}^{-}\right)\right)\right\| \\
\longrightarrow 0, \quad \text { as } t \rightarrow s
\end{gathered}
$$

by the strong continuity of $U(t, s)$ and the compactness of

$$
U((1 / m), 0) I_{i}\left(u\left(t_{i}^{-}\right)\right) \quad(i=1, \ldots, n) \quad \text { in } X .
$$

Consequently, we have proved, noticing Lemma 3.4, that $\Gamma_{m}$ is compact on $\Omega_{k_{0}}$. This enables us to deduce that $\Gamma_{m}$ has at least one fixed point $u_{m} \in \Omega_{k_{0}}$ for each $m \in \Theta$. The proof is then completed. 
Suppose that $u_{m}$, coming from Lemma 3.5, is a fixed point of $\Gamma_{m}$ corresponding to $m \in \Theta$. Then, from Lemma 3.5, it follows that there exists a $k_{0}>0$ such that $u_{m} \in \Omega_{k_{0}}$ for all $m \in \Theta$ and $u_{m}$ satisfies the integral equation

$$
\begin{aligned}
u_{m}(t)= & U(t, 0) U\left(\frac{1}{m}, 0\right) H\left(u_{m}\right)+\int_{0}^{t} U(t, s) F\left(s, u_{m}(s)\right) \mathrm{d} s \\
& +\sum_{0<t_{i}<t} U\left(t, t_{i}\right) U\left(\frac{1}{m}, 0\right) I_{i}\left(u_{m}\left(t_{i}^{-}\right)\right), \quad t \in[0, T], m \in \Theta .
\end{aligned}
$$

Now, write

$$
\begin{aligned}
& \left(\Gamma^{2} u_{m}\right)(t)=U(t, 0) U\left(\frac{1}{m}, 0\right) H\left(u_{m}\right), \\
& \left(\Gamma^{3} u_{m}\right)(t)=\sum_{0<t_{i}<t} U\left(t, t_{i}\right) U\left(\frac{1}{m}, 0\right) I_{i}\left(u_{m}\left(t_{i}^{-}\right)\right),
\end{aligned}
$$

and let $\mu \in(0, \eta)$ be fixed with $\eta$ being the constant in $\left(\mathrm{H}_{3}\right)$ (ii).

Lemma 3.6. Under the hypotheses of Lemma 3.5, $\left\{\Gamma^{2} u_{m} ; m \in \Theta\right\}_{\left[\mu, t_{1}\right]}$ is precompact in $C\left(\left[\mu, t_{1}\right] ; X\right),\left\{\Gamma^{2} u_{m} ; m \in \Theta\right\}_{\bar{J}_{i}}$ for each $i=1, \ldots, n$ is precompact in $C\left(\bar{J}_{i} ; X\right)$, and $\left\{\Gamma^{i} u_{m} ; m \in \Theta\right\}$ for $i=1,3$ is precompact in $P C([0, T] ; X)$.

Proof. From the compactness of $U(t, 0)(t \in[\mu, T])$ in $X$, the boundedness of $U((1 / m), 0)$, Remark 3.2, and $\left(\mathrm{H}_{3}\right)$ (i), it is not difficult to see that $\left\{U(t, 0) U((1 / m), 0) H\left(u_{m}\right) ; m \in \Theta\right\}$ for each $t \in\left[\mu, t_{1}\right]$ is precompact in $X$, and for $s_{1}, s_{2} \in\left[\mu, t_{1}\right]$ with $s_{1} \leq s_{2}$,

$$
\begin{aligned}
\left\|U\left(s_{2}, 0\right) U\left(\frac{1}{m}, 0\right) H\left(u_{m}\right)-U\left(s_{1}, 0\right) U\left(\frac{1}{m}, 0\right) H\left(u_{m}\right)\right\| \\
=\left\|\left(U\left(s_{2}, 0\right)-U\left(s_{1}, 0\right)\right) U\left(\frac{1}{m}, 0\right) H\left(u_{m}\right)\right\| \\
\longrightarrow 0, \quad \text { as } s_{2} \rightarrow s_{1},
\end{aligned}
$$

uniformly for $m \in \Theta$. Hence, an application of Arzela-Ascoli's theorem justifies that $\left\{\Gamma^{2} u_{m} ; m \in \Theta\right\}_{\left[\mu, t_{1}\right]}$ is precompact in $C\left(\left[\mu, t_{1}\right] ; X\right)$. The 
same idea can be used to prove that, for each $i=1, \ldots, n,\left\{\Gamma^{2} u_{m} ; m \in\right.$ $\Theta\}_{\bar{J}_{i}}$ is precompact in $C\left(\bar{J}_{i} ; X\right)$.

Next, we treat $\left\{\Gamma^{1} u_{m} ; m \in \Theta\right\}$. Let $t \in\left(0, t_{1}\right]$ be fixed. For any $\varepsilon \in(0, t)$, note that $U(t, t-\varepsilon / 2) \in \mathscr{L}(X)$ and $U(t-\varepsilon / 2, t-\varepsilon)$ is compact in $X$ by $\left(H_{1}\right)$. Therefore, as

$$
\begin{aligned}
\left(\Gamma^{1} u_{m}\right)(t)= & \int_{0}^{t} U(t, s) F\left(s, u_{m}(s)\right) \mathrm{d} s \\
= & \int_{t-\varepsilon}^{t} U(t, s) F\left(s, u_{m}(s)\right) \mathrm{d} s \\
& +U\left(t, t-\frac{\varepsilon}{2}\right) U\left(t-\frac{\varepsilon}{2}, t-\varepsilon\right) \\
& \times \int_{0}^{t-\varepsilon} U(t-\varepsilon, s) F\left(s, u_{m}(s)\right) \mathrm{d} s,
\end{aligned}
$$

and

$$
\left\|\int_{t-\varepsilon}^{t} U(t, s) F\left(s, u_{m}(s)\right) \mathrm{d} s\right\| \leq M \int_{t-\varepsilon}^{t} f_{k_{0}}(s) \mathrm{d} s \longrightarrow 0 \quad \text { as } \varepsilon \rightarrow 0,
$$

uniformly for $m \in \Theta$ due to $\left(\mathrm{H}_{2}\right)$, we conclude, using total boundedness, that, for each $t \in\left(0, t_{1}\right],\left\{\left(\Gamma^{1} u_{m}\right)(t) ; m \in \Theta\right\}$ is relatively compact in $X$. Let $\delta>0$ be small enough. Furthermore, for the case when $0<s_{1}<s_{2} \leq t_{1}$, in view of $\left(\mathrm{H}_{2}\right)$ and Remark 3.2, we have

$$
\begin{aligned}
&\left\|\left(\Gamma^{1} u_{m}\right)\left(s_{2}\right)-\left(\Gamma^{1} u_{m}\right)\left(s_{1}\right)\right\| \\
& \leq \int_{s_{1}}^{s_{2}}\left\|U\left(s_{2}, s\right) F\left(s, u_{m}(s)\right)\right\| \mathrm{d} s \\
&+\int_{0}^{s_{1}-\delta}\left\|\left(U\left(s_{2}, s\right)-U\left(s_{1}, s\right)\right) F\left(s, u_{m}(s)\right)\right\| \mathrm{d} s \\
&+\int_{s_{1}-\delta}^{s_{1}}\left\|\left(U\left(s_{2}, s\right)-U\left(s_{1}, s\right)\right) F\left(s, u_{m}(s)\right)\right\| \mathrm{d} s \\
& \leq M \int_{s_{1}}^{s_{2}} f_{k_{0}}(s) \mathrm{d} s \\
&+\sup _{s \in\left[0, s_{1}-\delta\right]}\left\|\left(U\left(s_{2}, s\right)-U\left(s_{1}, s\right)\right)\right\|_{\mathscr{L}(X)} \int_{0}^{s_{1}-\delta} f_{k_{0}}(s) \mathrm{d} s
\end{aligned}
$$




$$
+2 M \int_{s_{1}-\delta}^{s_{1}} f_{k_{0}}(s) \mathrm{d} s \longrightarrow 0 \quad \text { as } s_{2}-s_{1} \rightarrow 0, \delta \rightarrow 0,
$$

uniformly for $m \in \Theta$. For the case when $0=s_{1}<s_{2} \leq t_{1}$, since

$$
\left\|\int_{0}^{s_{2}} U\left(s_{2}, s\right) F\left(s, u_{m}(s)\right) \mathrm{d} s\right\| \leq M \int_{0}^{s_{2}} f_{k_{0}}(s) \mathrm{d} s,
$$

in view of $\left(\mathrm{H}_{2}\right),\left\|\left(\Gamma^{1} u_{m}\right)\left(t_{2}\right)\right\|$ can be made small when $s_{2}$ is small independently of $u_{m}(m \in \Theta)$. Thus, we verify that $\left.\left\{\Gamma^{1} u_{m} ; m \in \Theta\right\}\right|_{J_{0}}$ is precompact in $C\left(J_{0} ; X\right)$, with the aid of Arzela-Ascoli's theorem. The same idea can be used to prove that, for each $i=1, \ldots, n$, $\left\{\Gamma^{1} u_{m} ; m \in \Theta\right\}_{\bar{J}_{i}}$ is precompact in $C\left(\bar{J}_{i} ; X\right)$. Accordingly, we obtain, thanks to Lemma 2.1, that

$$
\left\{\Gamma^{1} u_{m} ; m \in \Theta\right\} \quad \text { is precompact in } P C([0, T] ; X) .
$$

As shown in the above arguments, $\left\{u_{m} ; m \in \Theta\right\}_{\left[\mu, t_{1}\right]}$ is precompact in $C\left(\left[\mu, t_{1}\right] ; X\right)$. Accordingly, one can assume, without loss of generality, that $u_{m} \rightarrow u_{0}$ in $C\left(\left[\mu, t_{1}\right] ; X\right)$ as $m \rightarrow \infty$, which implies in particular that $u_{m}\left(t_{1}\right) \rightarrow u\left(t_{1}\right)$ in $X$ as $m \rightarrow \infty$. So, in view of the continuity of $I_{1}$, we obtain $I_{1}\left(u_{m}\left(t_{1}\right)\right) \rightarrow I_{1}\left(u\left(t_{1}\right)\right)$ in $X$ as $m \rightarrow \infty$. That is, $\left\{I_{1}\left(u_{m}\left(t_{1}\right)\right) ; m \in \Theta\right\}$ is relatively compact in $X$. This, together with the strong continuity of $U(t, s)$ gives that

$$
\begin{aligned}
& \left\|I_{1}\left(u\left(t_{1}\right)\right)-U\left(\frac{1}{m}, 0\right) I_{1}\left(u_{m}\left(t_{1}\right)\right)\right\| \\
& \leq\left\|U\left(\frac{1}{m}, 0\right)\left(I_{1}\left(u\left(t_{1}\right)\right)-I_{1}\left(u_{m}\left(t_{1}\right)\right)\right)\right\| \\
& \quad+\left\|I_{1}\left(u\left(t_{1}\right)\right)-U\left(\frac{1}{m}, 0\right) I_{1}\left(u\left(t_{1}\right)\right)\right\| \\
& \leq \quad M\left\|I_{1}\left(u\left(t_{1}\right)\right)-I_{1}\left(u_{m}\left(t_{1}\right)\right)\right\| \\
& \quad+\left\|I_{1}\left(u\left(t_{1}\right)\right)-U\left(\frac{1}{m}, 0\right) I_{1}\left(u\left(t_{1}\right)\right)\right\| \longrightarrow 0 \text { as } m \rightarrow \infty .
\end{aligned}
$$

Hence, we deduce that

$$
\left\{U\left(\frac{1}{m}, 0\right) I_{1}\left(u_{m}\left(t_{1}\right)\right) ; m \in \Theta\right\} \text { is relatively compact in } X \text {. }
$$

So $\left.\left\{U\left(t, t_{1}\right) U((1 / m), 0) I_{1}\left(u_{m}\left(t_{1}^{-}\right)\right) ; m \in \Theta\right\}\right|_{\left(t_{1}, t_{2}\right]}$ is also relatively compact due to the compactness of $U\left(t, t_{1}\right)$ for $t>t_{1}$. At the same time, 
from (3.3), it follows that for $s_{1}, s_{2} \in \bar{J}_{1}$ with $s_{1} \leq s_{2}$,

$$
\begin{aligned}
& \left\|U\left(s_{2}, t_{1}\right) U\left(\frac{1}{m}, 0\right) I_{1}\left(u_{m}\left(t_{1}^{-}\right)\right)-U\left(s_{1}, t_{1}\right) U\left(\frac{1}{m}, 0\right) I_{1}\left(u_{m}\left(t_{1}^{-}\right)\right)\right\| \\
& =\left\|\left[U\left(s_{2}, t_{1}\right)-U\left(s_{1}, t_{1}\right)\right] U\left(\frac{1}{m}, 0\right) I_{1}\left(u\left(t_{1}^{-}\right)\right)\right\| \longrightarrow 0, \quad \text { as } s_{2} \rightarrow s_{1},
\end{aligned}
$$

uniformly for $m \in \Theta$. Therefore, we find that

$$
\left\{\left.U\left(t, t_{1}\right) U\left((1 / m, 0) I_{1}\left(u_{m}\left(t_{1}^{-}\right)\right) ; m \in \Theta\right\}\right|_{\bar{J}_{1}}\right.
$$

is precompact in $C\left(\bar{J}_{1} ; X\right)$ in view of the Arzela-Ascoli theorem. A similar argument enables us to conclude that, for each $i=2, \ldots, n$, $\left.\left\{U\left(t, t_{i}\right) U((1 / m), 0) I_{i}\left(u_{m}\left(t_{i}^{-}\right)\right) ; m \in \Theta\right\}\right|_{\bar{J}_{i}}$ is precompact in $C\left(\bar{J}_{i} ; X\right)$. Accordingly, we deduce, thanks to Lemma 2.1, that

$$
\left\{\Gamma^{3} u_{m} ; m \in \Theta\right\} \quad \text { is precompact in } P C([0, T] ; X) .
$$

The proof is completed.

Lemma 3.7. Let the hypotheses $\left(\mathrm{H}_{1}\right)-\left(\mathrm{H}_{4}\right)$ hold. Then $\left\{\Gamma^{2} u_{m} ; m \in\right.$ $\Theta\}\left.\right|_{[0, \eta]}$ is precompact in $C([0, \eta] ; X)$.

Proof. From Lemma 3.6 it follows readily that $\left\{u_{m} ; m \in \Theta\right\}_{\left[\mu, t_{1}\right]}$ is precompact in $C\left(\left[\mu, t_{1}\right] ; X\right)$ and $\left\{u_{m} ; m \in \Theta\right\}_{\bar{J}_{i}}$ for each $i=1, \ldots, n$ is precompact in $C\left(\bar{J}_{i} ; X\right)$. Write

$$
\bar{u}_{m}(t)= \begin{cases}u_{m}(t) & \text { if } t \in[\eta, T], \\ u_{m}(\eta) & \text { if } t \in[0, \eta] .\end{cases}
$$

It is clear that $H\left(u_{m}\right)=H\left(\bar{u}_{m}\right)$ in view of $\left(\mathrm{H}_{3}\right)$ (ii). Also, without loss of generality, we may assume that $\bar{u}_{m} \rightarrow u$ in $P C(0, T ; X)$ as $m \rightarrow \infty$. This, together with the continuity of $H$ and the strong continuity of $U(t, s)$, gives that, for any $t \in[0, \eta]$,

$$
\begin{aligned}
& \left\|U(t, 0) H(u)-U(t, 0) U\left(\frac{1}{m}, 0\right) H\left(u_{m}\right)\right\| \\
& =\left\|U(t, 0) H(u)-U(t, 0) U\left(\frac{1}{m}, 0\right) H\left(\bar{u}_{m}\right)\right\|
\end{aligned}
$$




$$
\begin{gathered}
\leq\left\|U(t, 0) H(u)-U(t, 0) U\left(\frac{1}{m}, 0\right) H(u)\right\| \\
+\left\|U(t, 0) U\left(\frac{1}{m}, 0\right)\left(H(u)-H\left(\bar{u}_{m}\right)\right)\right\| \\
\leq M\left\|H(u)-U\left(\frac{1}{m}, 0\right) H(u)\right\|+M^{2}\left\|H(u)-H\left(\bar{u}_{m}\right)\right\| \\
\longrightarrow 0 \text { as } m \rightarrow \infty,
\end{gathered}
$$

from which we see that the set $\left\{U(t, 0) U((1 / m), 0) H\left(u_{m}\right) ; m \in \Theta\right\}$ for any $t \in[0, \eta]$ is relatively compact in $X$. This means, in particular, that $\left\{U((1 / m), 0) H\left(u_{m}\right) ; m \in \Theta\right\}$ is relatively compact in $X$, which, together with the strong continuity of $U(t, s)$, yields that for $s_{1}, s_{2} \in$ $[0, \eta]$ with $s_{1} \leq s_{2}$,

$$
\begin{aligned}
& \left\|U\left(s_{2}, 0\right) U\left(\frac{1}{m}, 0\right) H\left(u_{m}\right)-U\left(s_{1}, 0\right) U\left(\frac{1}{m}, 0\right) H\left(u_{m}\right)\right\| \\
& \quad \leq\left\|\left(U\left(s_{2}, 0\right)-U\left(s_{1}, 0\right)\right) U\left(\frac{1}{m}, 0\right) H\left(u_{m}\right)\right\| \rightarrow 0 \quad \text { as } s_{2} \rightarrow s_{1},
\end{aligned}
$$

uniformly for $m \in \Theta$. Consequently, we conclude that the assertion of the lemma holds due to Arzela-Ascoli's theorem. This completes the proof.

Now, we can state our main result of this section.

Theorem 3.8. Let the hypotheses $\left(\mathrm{H}_{1}\right)-\left(\mathrm{H}_{4}\right)$ hold. Then the Cauchy problem (1.1) has at least one mild solution provided that

$$
M \sigma+M^{2}\left(\mu+\sum_{i=1}^{n} \gamma_{i}\right)<1 .
$$

Remark 3.9. Note that, in Theorem 3.8, the impulsive item and nonlocal item only verify the continuity and the growth conditions.

Proof of Theorem 3.8. Let $u_{m}$, coming from Lemma 3.5, be a fixed point of $\Gamma_{m}$ corresponding to $m \in \Theta$. Combining Lemma 3.6 and 
Lemma 3.7, one can see, thanks to Lemma 2.1, that

$$
\left\{u_{m} ; m \in \Theta\right\} \text { is precompact in } P C([0, T] ; X),
$$

which implies that there is a subsequence of $\left\{u_{m} ; m \in \Theta\right\}$, again denoted by $\left\{u_{m}\right\}$, and a $u \in P C(0, T ; X)$ such that $u_{m} \rightarrow u$ in $P C(0, T ; X)$ as $m \rightarrow \infty$. Note that $u_{m} \in P C(0, T ; X)$ satisfies the integral equation (3.2). Letting $m \rightarrow \infty$ on both sides of (3.2), one finds, noticing the continuity of $H, I_{i}(i=1, \ldots, n)$, and $F$ with respect to the second argument, that $u$ is a mild solution of Cauchy problem (1.1). This completes the proof.

The following corollaries are generalizations of Theorem 3.8.

Corollary 3.10. Under the hypotheses $\left(\mathrm{H}_{1}\right),\left(\mathrm{H}_{2}\right)$ and $\left(\mathrm{H}_{4}\right)$, for every $u_{0} \in X$, the non-autonomous impulsive Cauchy problem

$$
\left\{\begin{array}{l}
u^{\prime}(t)=A(t) u(t)+F(t, u(t)), \quad t \in[0, T] \backslash\left\{t_{1}, \ldots, t_{n}\right\}, \\
\Delta u\left(t_{i}\right)=I_{i}\left(u\left(t_{i}\right)\right), \quad i=1, \ldots, n, \\
u(0)=u_{0},
\end{array}\right.
$$

has at least one mild solution, provided that $M \sigma+M^{2} \sum_{i=1}^{n} \gamma_{i}<1$.

Corollary 3.11. Assume that hypotheses $\left(\mathrm{H}_{1}\right),\left(\mathrm{H}_{2}\right)$ and $\left(\mathrm{H}_{4}\right)$ are satisfied. Then the non-autonomous impulsive Cauchy problem with nonlocal initial condition

$$
\left\{\begin{array}{l}
u^{\prime}(t)=A(t) u(t)+F(t, u(t)), \quad t \in[0, T] \backslash\left\{t_{1}, \ldots, t_{n}\right\}, \\
\Delta u\left(t_{i}\right)=I_{i}\left(u\left(t_{i}\right)\right), \quad i=1, \ldots, n, \\
u(0)=\sum_{i=1}^{p} C_{i} u\left(s_{i}\right), \quad 0<s_{1}<\cdots<s_{p-1}<s_{p}<T,
\end{array}\right.
$$

where $C_{i}(i=1, \ldots, p)$ are given constants, has at least one mild solution provided that $M \sigma+M^{2}\left(\sum_{i=1}^{p}\left|C_{i}\right|+\sum_{i=1}^{n} \gamma_{i}\right)<1$.

Proof. Define

$$
H(u)=\sum_{i=1}^{p} C_{i} u\left(s_{i}\right), \quad u \in P C([0, T] ; X) .
$$

Then it follows readily that function $H$ satisfies the hypothesis $\left(\mathrm{H}_{2}\right)$ with $\Phi(r)=r \sum_{i=1}^{p}\left|C_{i}\right|$ and $\mu=\sum_{i=1}^{p}\left|C_{i}\right|$. Hence, the conclusion holds due to Theorem 3.8. The proof is completed. 
4. Cauchy problems of neutral type. In this section, we extend the results obtained in Section 3 to the Cauchy problem of neutral type (1.2).

It follows from (c) that there exist constants $M_{1}>0,0<\alpha \leq 1$, such that

$$
\left\|A(t) A^{-1}(0)\right\|_{\mathscr{L}(X)} \leq M_{1} T^{\alpha}
$$

for all $t \in[0, T]$. Let $X^{1}$ denote the Banach space $D$ endowed with the graph norm $\|u\|_{1}=\|A(0) u\|$ for $u \in X^{1}$.

Definition 4.1. A mild solution to Cauchy problem (1.2) is a function $u \in P C([0, T] ; X)$ satisfying the integral equation

$$
\begin{aligned}
u(t)= & U(t, 0)[H(u)-g(0, u(0))]+g(t, u(t)) \\
& +\int_{0}^{t} U(t, \tau) A(\tau) g(\tau, u(\tau)) \mathrm{d} \tau \\
& +\int_{0}^{t} U(t, \tau) F(\tau, u(\tau)) \mathrm{d} \tau \\
& +\sum_{0<t_{i}<t} U\left(t, t_{i}\right) I_{i}\left(u\left(t_{i}^{-}\right)\right), \quad t \in[0, T] .
\end{aligned}
$$

Remark 4.2. It will be seen later that the integrals on right side in (4.2), being ones in sense of Bochner (see [25]), are reasonable.

Assume that

$\left(\mathrm{H}_{5}\right)$ (i) The function $g:[0, T] \times X \rightarrow X^{1}$ is continuous and $g(\cdot, u(\cdot))=$ $g(\cdot, w(\cdot))$ for any $u, w \in P C([0, T] ; X)$ with $u(t)=w(t)(t \in$ $[\eta, T])$.

(ii) There exist a constant $L_{g}$ and a nondecreasing function $\Upsilon: \mathbf{R}^{+} \rightarrow \mathbf{R}^{+}$such that

$$
\begin{aligned}
\|g(t, u)-g(t, v)\|_{1} & \leq L_{g}\|u-v\|, \\
\|g(t, u)\|_{1} & \leq \Upsilon(\|u\|)
\end{aligned}
$$


for all $t \in[0, T], u, v \in X$ and

$$
\liminf _{r \rightarrow+\infty} \frac{\Upsilon(r)}{r}=\sigma_{1}
$$

Our main result in this section is the following theorem.

Theorem 1. If assumptions $\left(\mathrm{H}_{1}\right)-\left(\mathrm{H}_{5}\right)$ are satisfied together with

$$
\begin{aligned}
M \sigma+ & M^{2}\left(\mu+\sum_{i=1}^{n} \gamma_{i}\right) \\
& +\left((M+1)\left\|A^{-1}(0)\right\|_{\mathscr{L}(X)}+M M_{1} T^{\alpha+1}\right) \max \left\{\sigma_{1}, L_{g}\right\}<1,
\end{aligned}
$$

then the Cauchy problem (1.2) admits at least one mild solution.

Proof. Fix $m \in \Theta$. Let us assume that the mapping $\Gamma_{m}$ is defined the same as in Section 3. Write, for $u \in P C([0, T] ; X)$,

$$
\begin{aligned}
\left(\Gamma^{4} u\right)(t)= & -U(t, 0) g(0, u(0))+g(t, u(t)) \\
& +\int_{0}^{t} U(t, \tau) A(\tau) g(\tau, u(\tau)) \mathrm{d} \tau \\
:= & -U(t, 0) g(0, u(0))+\left(\Gamma_{g}^{4} u\right)(t), \quad t \in[0, T] .
\end{aligned}
$$

It is clear that $\Gamma^{4}$, mapping $P C(0, T ; X)$ into itself, is well defined. Also, from the proof of Lemma 3.5, $\left(\mathrm{H}_{5}\right)$ (ii) and (4.1), we observe that, for $t \in[0, T]$ and $u, v \in \Omega_{r}$,

$$
\begin{aligned}
\left\|\left(\Gamma_{m} u+\Gamma^{4} v\right)(t)\right\| \leq & M^{2} \Phi(r)+M \int_{0}^{t} f_{r}(s) \mathrm{d} s+M^{2} \sum_{i=1}^{n} \Psi_{i}(r) \\
& +\left((M+1)\left\|A^{-1}(0)\right\|_{\mathscr{L}(X)}+M M_{1} T^{\alpha+1}\right) \Upsilon(r),
\end{aligned}
$$

which, together with (4), yields that there exists a $k_{0}>0$ such that $\Gamma_{m} u+\Gamma^{4} v$ maps $\Omega_{k_{0}}$ into itself for every pair $u, v \in \Omega_{k_{0}}$. Moreover, one finds that $\Gamma^{4}$ is a contraction on $\Omega_{k_{0}}$. In fact, this can be seen from 
(4) and

$$
\begin{aligned}
\left\|\left(\Gamma^{4} u-\Gamma^{4} v\right)(t)\right\| \leq & M L_{g}\left\|A^{-1}(0)\right\|_{\mathscr{L}(X)}\|u(0)-v(0)\| \\
& +L_{g}\left\|A^{-1}(0)\right\|_{\mathscr{L}(X)}\|u(t)-v(t)\| \\
& +M M_{1} T^{\alpha} L_{g} \int_{0}^{t}\|u(\tau)-v(\tau)\| \mathrm{d} \tau \\
\leq & \left((M+1) L_{g}\left\|A^{-1}(0)\right\|_{\mathscr{L}(X)}+M M_{1} L_{g} T^{\alpha+1}\right)\|u-v\|_{P C}
\end{aligned}
$$

for $t \in[0, T]$ and $u, v \in \Omega_{k_{0}}$. At the same time, as proved in Lemma 3.5, $\Gamma_{m}$ is continuous and compact on $\Omega_{k_{0}}$.

We see, from the arguments above, that $\Gamma_{m}+\Gamma^{4}$ verifies all conditions of Lemma 2.3, which enables us to deduce that $\Gamma_{m}+\Gamma^{4}$ has at least one fixed point $v_{m} \in \Omega_{k_{0}}$ for every $m \in \Theta$, that is, $v_{m}$ satisfies the integral equation

$$
\begin{aligned}
v_{m}(t)= & U(t, 0)\left[U\left(\frac{1}{m}, 0\right) H\left(v_{m}\right)-g\left(0, u_{m}(0)\right)\right]+g\left(t, v_{m}(t)\right) \\
& +\int_{0}^{t} U(t, \tau) A(\tau) g\left(\tau, v_{m}(\tau)\right) \mathrm{d} \tau \\
& +\int_{0}^{t} U(t, \tau) F\left(\tau, v_{m}(\tau)\right) \mathrm{d} \tau \\
& +\sum_{0<t_{i}<t} U\left(t, t_{i}\right) U\left(\frac{1}{m}, 0\right) I_{i}\left(v_{m}\left(t_{i}^{-}\right)\right), \quad t \in[0, T] .
\end{aligned}
$$

Now, consider the set $\left\{v_{m} ; m \in \Theta\right\}$. Let $\mu^{\prime} \in(0, \eta)$ be fixed with $\eta$ being the constant in $\left(\mathrm{H}_{3}\right)$ (ii) and $\left(\mathrm{H}_{5}\right)$ (i), and assume that the mappings $\Gamma^{i}(i=1,2,3)$ are defined the same as in Section 3. Note that

$$
\begin{gathered}
v_{m}(t)=\left(\Gamma^{1} v_{m}\right)(t)+\left(\Gamma^{2} v_{m}\right)(t)+\left(\Gamma^{3} v_{m}\right)(t)+\left(\Gamma^{4} v_{m}\right)(t), \\
t \in[0, T], \quad m \in \Theta .
\end{gathered}
$$

From the compactness of $U(t, 0)$ for $t \in(0, T]$ and $\left(\mathrm{H}_{5}\right)$ (ii), it follows readily that $\left.\left\{U(t, 0) g\left(0, v_{m}(0)\right) ; m \in \Theta\right\}\right|_{\left[\mu^{\prime}, t_{1}\right]}$ is precompact in $C\left(\left[\mu^{\prime}, t_{1}\right] ; X\right)$ and $\left\{U(t, 0) g\left(0, v_{m}(0)\right) ; m \in \Theta\right\}_{\bar{J}_{i}}$ for each $i=1, \ldots, n$ is precompact in $C\left(\bar{J}_{i} ; X\right)$. Also, by Lemma 3.6 we note that $\left\{\Gamma^{2} v_{m} ; m \in\right.$ $\Theta\}_{\left[\mu^{\prime}, t_{1}\right]}$ is precompact in $C\left(\left[\mu^{\prime}, t_{1}\right] ; X\right),\left\{\Gamma^{2} v_{m} ; m \in \Theta\right\}_{\bar{J}_{i}}$ for each $i=$ 
$1, \ldots, n$ is precompact in $C\left(\bar{J}_{i} ; X\right)$ and $\left\{\Gamma^{1} v_{m} ; m \in \Theta\right\}$ is precompact in $P C([0, T] ; X)$. Denote

$$
\widetilde{u}(t)= \begin{cases}u(t) & \text { if } t \in\left[\mu^{\prime}, T\right] \\ u\left(\mu^{\prime}\right) & \text { if } t \in\left[0, \mu^{\prime}\right]\end{cases}
$$

for $u \in C\left(\left[\mu^{\prime}, t_{1}\right] ; X\right)$. Therefore, for $u, v \in C\left(\left[\mu^{\prime}, t_{1}\right] ; X\right)$ and $t \in\left[\mu^{\prime}, t_{1}\right]$, we have

$$
\begin{aligned}
\left\|\left(\Gamma_{g}^{4} u\right)(t)-\left(\Gamma_{g}^{4} v\right)(t)\right\| \leq & \left\|A^{-1}(0)\right\|_{\mathscr{L}(X)}\|g(t, u(t))-g(t, v(t))\|_{1} \\
& +M M_{1} T^{\alpha} \int_{0}^{t}\|g(\tau, \widetilde{u}(\tau))-g(\tau, \widetilde{v}(\tau))\|_{1} \mathrm{~d} \tau \\
\leq & L_{g}\left\|A^{-1}(0)\right\| \mathscr{L}(X)\|u(t)-v(t)\| \\
& +M M_{1} L_{g} T^{\alpha+1} \sup _{0 \leq t \leq t_{1}}\|\widetilde{u}(t)-\widetilde{v}(t)\| \\
\leq & \left(L_{g}\left\|A^{-1}(0)\right\| \mathscr{L}(X)+M M_{1} L_{g} T^{\alpha+1}\right) \\
& \times \sup _{\mu^{\prime} \leq t \leq t_{1}}\|u(t)-v(t)\|,
\end{aligned}
$$

which gives that $\Gamma_{g}^{4}$, mapping $C\left(\left[\mu^{\prime}, t_{1}\right] ; X\right)$ into itself, is Lipschitz continuous. Hence, one has

$$
\begin{aligned}
\beta\left(\left.\left\{v_{m} ; m \in \Theta\right\}\right|_{\left[\mu^{\prime}, t_{1}\right]}\right) \leq & \beta\left(\left\{\Gamma^{1} v_{m} ; m \in \Theta\right\}_{\left[\mu^{\prime}, t_{1}\right]}\right) \\
& +\beta\left(\left\{\Gamma^{2} v_{m} ; m \in \Theta\right\}_{\left[\mu^{\prime}, t_{1}\right]}\right) \\
& +\beta\left(\left.\left\{U(t, 0) g\left(0, v_{m}(0)\right) ; m \in \Theta\right\}\right|_{\left[\mu^{\prime}, t_{1}\right]}\right) \\
& +\beta\left(\left\{\Gamma^{4} v_{m} ; m \in \Theta\right\}_{\left[\mu^{\prime}, t_{1}\right]}\right) \\
\leq & \beta\left(\left\{\Gamma_{g}^{4} v_{m} ; m \in \Theta\right\}_{\left[\mu^{\prime}, t_{1}\right]}\right) \\
\leq & \left(L_{g}\left\|A^{-1}(0)\right\|_{\mathscr{L}(X)}+M M_{1} L_{g} T^{\alpha+1}\right) \\
& \times \beta\left(\left.\left\{v_{m} ; m \in \Theta\right\}\right|_{\left[\mu^{\prime}, t_{1}\right]}\right),
\end{aligned}
$$

where $\beta(\cdot)$ stands for the Hausdorff measure of noncompactness (see [5]), from which together with (4), we see that $\beta\left(\left.\left\{v_{m} ; m \in \Theta\right\}\right|_{\left[\mu^{\prime}, t_{1}\right]}\right)=$ 0 . This proves that $\left.\left\{v_{m} ; m \in \Theta\right\}\right|_{\left[\mu^{\prime}, t_{1}\right]}$ is precompact in $C\left(\left[\mu^{\prime}, t_{1}\right] ; X\right)$.

The same idea as the last part of the proof in Lemma 3.6 can be used to prove that $\left\{v_{m} ; m \in \Theta\right\}_{\bar{J}_{i}}$ is precompact in $C\left(\bar{J}_{i} ; X\right)$. Furthermore, by applying a similar argument as that in Lemma 3.7, we have that $\left.\left\{\Gamma^{2} v_{m} ; m \in \Theta\right\}\right|_{[0, \eta]}$ is precompact in $C([0, \eta] ; X)$. 
As proven in the above arguments, we obtain

$$
\begin{aligned}
& \beta\left(\left\{v_{m} ; m \in \Theta\right\} \mid\right) \\
& \quad \leq\left((M+1) L_{g}\left\|A^{-1}(0)\right\|_{\mathscr{L}(X)}+M M_{1} L_{g} T^{\alpha+1}\right) \beta\left(\left\{v_{m} ; m \in \Theta\right\} \mid\right),
\end{aligned}
$$

which, together with (4), implies that

$$
\left\{v_{m} ; m \in \Theta\right\} \quad \text { is precompact in } P C([0, T] ; X) \text {. }
$$

Moreover, following from the same idea as the proof in Theorem 3.8, we obtain that the theorem remains true. This completes the proof.

5. An example. To illustrate our abstract results, in this section let us consider a system of partial differential equations with impulsive and nonlocal initial conditions, which does not aim at generality but indicates how our theorem can be applied to a concrete problem. Such an example is inspired directly from the work of Fan and $\mathrm{Li}[\mathbf{1 5}$, Example 6.2] and Wang and Yang [30, Example 4.1].

Consider the following system

$$
\left\{\begin{array}{l}
\frac{\partial u(t, x)}{\partial t}=\frac{\partial^{2} u(t, x)}{\partial x^{2}}-a(t) u(t, x)+b(t) u(t, x) \sin u^{2}(t, x), \\
t \in[0, T] \backslash\left\{t_{1}, \ldots, t_{n}\right\}, x \in[0, \pi], \\
u\left(t_{i}^{+}, x\right)-u\left(t_{i}^{-}, x\right)=\frac{u^{1 / 3}\left(t_{i}^{-}, x\right)}{1+\left|u\left(t_{i}^{-}, x\right)\right|}, \quad x \in[0, \pi], \quad i=1, \ldots, n, \\
u(0, x)=u_{0}(x)+\sum_{i=1}^{p} C_{i} u^{1 / 3}\left(s_{i}, x\right), \quad x \in[0, \pi], 0<s_{1}<\cdots<s_{p}<T,
\end{array}\right.
$$

supplemented with homogeneous Dirichlet boundary condition $u(t, 0)=$ $u(t, \pi)=0(t \in[0, T])$, where $0<t_{1}<t_{2}<\cdots<t_{n}<T$ are pre-fixed numbers. We assume that

$\left(\mathrm{H}_{a}\right) a:[0, T] \rightarrow \mathbf{R}$ is a continuously differentiable function and

$$
a_{\min }:=\min _{t \in[0, T]} a(t)>-1 .
$$

$\left(\mathrm{H}_{b}\right) \quad b \in L^{1}\left(0, T ; \mathbf{R}^{+}\right)$and $\|b\|_{L^{1}(0, T)}<1$.

Here, our objective is to show the existence of mild solutions to system (5.1). 
Take $X=L^{2}[0, \pi]$ with the norm $\|\cdot\|_{L^{2}[0, \pi]}$ and inner product $(\cdot, \cdot)_{2}$. Define an operator $B: D(B) \subset X \rightarrow X$ by

$$
\begin{aligned}
B u & =\frac{\partial^{2}}{\partial x^{2}} u, \quad u \in D(B), \\
D(B) & =\left\{u \in X ; u, u^{\prime} \quad\right. \text { are absolutely continuous, } \\
& \left.u^{\prime \prime} \in X, \text { and } u(0)=u(\pi)=0\right\} .
\end{aligned}
$$

It is well known that $B$ has a discrete spectrum, and its eigenvalues are $-n^{2}, n \in \mathbf{N}^{+}$with the corresponding normalized eigenvectors $y_{n}(x)=\sqrt{2 / \pi} \sin (n x)$. More details about these facts can be seen from the monograph [27] of Pazy.

Put

$$
\begin{aligned}
D(A(t)) & =D(B), \quad t \in[0, T], \\
A(t) u & =B u-a(t) u, \quad u \in D(A(t)) .
\end{aligned}
$$

Then, from our hypotheses, it is clear that $(A(t))_{t \in[0, T]}$ satisfies conditions (a) $-(\mathrm{c})$, which ensures that it generates an evolution family $\{U(t, s)\}_{0 \leq s \leq t \leq T}$ :

$$
\begin{gathered}
U(t, s) u=\sum_{n=1}^{\infty} e^{-\left(\int_{s}^{t} a(\tau) d \tau+n^{2}(t-s)\right)}\left(u, y_{n}\right)_{2} y_{n} \\
\quad \text { for } 0 \leq s \leq t \leq T, u \in X .
\end{gathered}
$$

A direct calculation gives

$$
\|U(t, s)\|_{\mathscr{L}(X)} \leq e^{-\left(1+a_{m i n}\right)(t-s)} \quad \text { for } 0 \leq s \leq t \leq T .
$$

Note also that, for each $t, s \in[0, T]$ with $t>s$, the operator $U(t, s)$ is a nuclear operator, which implies the compactness of $U(t, s)$ for $t>s$.

Define

$$
\begin{aligned}
u(t)(x) & =u(t, x), \\
F(t, u(t))(x) & =b(t) u(t, x) \sin u^{2}(t, x), \\
I_{i}\left(u\left(t_{i}\right)\right)(x) & =\frac{u^{1 / 3}\left(t_{i}^{-}, x\right)}{1+\left|u\left(t_{i}^{-}, x\right)\right|}, \\
H(u)(x) & =u_{0}(x)+\sum_{i=1}^{p} C_{i} u^{1 / 3}\left(s_{i}, x\right) .
\end{aligned}
$$


Note that system (5.1) can be reformulated as the abstract Cauchy problem (1.1) and the hypotheses $\left(\mathrm{H}_{1}\right)-\left(\mathrm{H}_{4}\right)$ hold with

$$
\begin{aligned}
f_{r}(t) & =b(t) r \\
\Psi_{i}(r) & =\pi^{1 / 3} r^{1 / 3}(i=1, \ldots, n), \\
\Phi(r) & =\left\|u_{0}\right\|_{L^{2}[0, \pi]}+\pi^{1 / 3} r^{1 / 3} \sum_{i=1}^{p}\left|C_{i}\right|, \\
\sigma & =\|b\|_{L^{1}(0, T)}, \\
\gamma_{i} & =0(i=1, \ldots, n), \\
\mu & =0, \quad M=1 .
\end{aligned}
$$

Hence, we deduce, under Hypotheses $\left(\mathrm{H}_{a}\right)$ and $\left(\mathrm{H}_{b}\right)$, that system (5.1) has at least one mild solution due to Theorem 3.8.

\section{REFERENCES}

1. N.U. Ahmed, Existence of optimal controls for a general class of impulsive systems on Banach space, SIAM J. Cont. Optim. 42 (2003), 669-685.

2. _ Optimal feedback control for impulsive systems on the space of finitely additive measures, Publ. Math. Debr. 70 (2007), 371-393.

3. A. Anguraj and K. Karthikeyan, Existence of solutions for impulsive neutral functional differential equations with nonlocal conditions, Nonlinear Anal. 70 (2009), 2717-2721.

4. D.D. Bainov and P.S. Simeonov, Systems with impulse effect, Ellis Horwood, Chichester, 1989.

5. J. Banas and K. Goebel, Measure of noncompactness in Banach spaces, Lect. Notes Pure Appl. Math. 60, Marcel Dekker, New York, 1980.

6. M. Benchohra, E.P. Gatsori, J. Henderson and S.K. Ntouyas, Nondensely defined evolution impulsive differential inclusions with nonlocal conditions, J. Math. Anal. Appl. 286 (2003), 307-325.

7. M. Benchohra, J. Henderson and S.K. Ntouyas, Impulsive differential equations and inclusions, vol. 2, Hindawi Publishing Corporation, New York, 2006.

8. L. Byszewski, Theorems about the existence and uniqueness of solutions of a semilinear evolution nonlocal Cauchy problem, J. Math. Anal. Appl. 162 (1991), 494-505.

9. T. Cardinali and P. Rubbioni, Impulsive semilinear differential inclusions: Topological structure of the solution set and solutions on non-compact domains, Nonlinear Anal. 69 (2008), 73-84.

10. P.J. Chen and M.E. Gurtin, On a theory of heat conduction involving two temperatures, Z. Angew. Math. Phys. 19 (1968), 614-627. 
11. K. Deng, Exponential decay of solutions of semilinear parabolic equations with nonlocal initial conditions, J. Math. Anal. Appl. 179 (1993), 630-637.

12. K.J. Engel and R. Nagel, One-parameter semigroups for linear evolution equations, GTM 194, Springer, Berlin, 2000.

13. K. Ezzinbi, X. Fu and K. Hilal, Existence and regularity in the $\alpha$-norm for some neutral partial differential equations with nonlocal conditions, Nonlinear Anal. 67 (2007), 1613-1622.

14. K. Ezzinbi and J. Liu, Nondensely defined evolution equations with nonlocal conditions, Math. Comp. Model. 36 (2002), 1027-1038.

15. Z.B. Fan and G. Li, Existence results for semilinear differential equations with nonlocal and impulsive conditions, J. Funct. Anal. 258 (2010), 1709-1727.

16. W.E. Fitzgibbon, Semilinear functional equations in Banach space, J. Differential Equations 29 (1978), 1-14.

17. X. Fu and K. Ezzinbi, Existence of solutions for neutral functional differential evolution equations with nonlocal conditions, Nonlinear Anal. 54 (2003), $215-227$.

18. D.J. Guo and X. Liu, Extremal solutions of nonlinear impulsive integrodifferential equations in Banach spaces, J. Math. Anal. Appl. 177 (1993), 538-552.

19. M.E. Hernández and S.M. Tanaka Aki, Global solutions for abstract impulsive differential equations, Nonlinear Anal. 72 (2010), 1280-1290.

20. V. Lakshmikantham, D.D. Bainov and P.S. Simeonov, Theory of impulsive differential equations, World Scientific, Singapore, 1989.

21. J. Liang, J.H. Liu and T.J. Xiao, Nonlocal impulsive problems for nonlinear diffrential equations in Banach spaces, Math. Comp. Model. 49 (2009), 798-804.

22. J. Liu, Nonlinear impulsive evolution equations, Dyn. Cont. Discr. Impul. Syst. 6 (1999), 77-85.

23. J. Liu, G. N'Gurkata and N. van Minh, A Massera type theorem for almost automorphic solutions of differential equations, J. Math. Anal. Appl. 299 (2004), 587-599.

24. X. Liu and A. Willms, Stability analysis and applications to large scale impulsive systems: A new approach, Canad. Appl. Math. Quart. 3 (1995), 419444 .

25. C.M. Marle, Mesures et probabilities, Hermann, Paris, 1974.

26. J.J. Nieto and D.O'Regan, Variational approach to impulsive differential equations, Nonlinear Anal. 10 (2009), 680-690.

27. A. Pazy, Semigroups of linear operators and applications to partial differential equations, in Applied mathematical sciences, vol. 44, Springer-Verlag, New York, 1983.

28. Y. Rogovchenko, Impulsive evolution systems: Main results and new trends, Dyn. Cont. Discr. Impul. Syst. 3 (1997), 57-88.

29. A.M. Samoilenko and N.A. Perestyuk, Impulsive differential equations, World Scientific, Singapore, 1995. 
30. R.N. Wang and Y.H. Yang, On the Cauchy problems of fractional evolution equations with nonlocal initial conditions, Results. Math. 2011, doi 10.1007/s00025011-0142-9.

31. K. Yosida, Functional analysis, 6th ed., Springer-Verlag, Berlin, 1980.

Department of Applied Mathematics, Guangdong University of Foreign Studies, Guangzhou 510421, P.R. China

Email address: rnwang@mail.ustc.edu.cn

Faculty of Sciences Semlalia, Department of Mathematics, Cadi Ayyad University, B.P. 2390, Marrakesh, Morocco

Email address: ezzinbi@uca.ma

Department of Mathematics, Nanchang University, Nanchang, Jiangxi 330031, P.R. CHINA

Email address: pxzhuncu@126.com 